\title{
A Survey Paper on E-Blood Bank and an Idea to use on Smartphone
}

\author{
Tushar Pandit \\ Dept. of I.T \\ Sinhgad Academy of \\ Engineering, Pune, \\ India.
}

\author{
Satish Niloor \\ Dept. of I.T \\ Sinhgad Academy of \\ Engineering, Pune, \\ India.
}

\author{
A.S. Shinde \\ Dept. of I.T \\ Sinhgad Academy of \\ Engineering, Pune, \\ India
}

\begin{abstract}
Blood is an important aspect for all living things. It proves to be a lifesaving component in case of emergency requirement. There are number of web based blood banks which are available for communication between blood bank and hospitals. None of the online blood bank offers the direct contact between donor and blood bank. This is the major drawback of the existing system. Existing systems are time consuming; require more man power and it is costly. This paper introduces comparison between existing system and improved system. The new idea will improve the existing system and it will move from conventional desktop system to mobile system. This paper introduces new features of improved system over existing system in many aspects. In this paper firstly we are going to explain merits and demerits of existing system. Also this paper will provide brief introduction about improved system and its features and Scope.
\end{abstract}

\section{General Terms}

Online E-blood bank, Global positioning system navigation.

\section{Keywords}

Blood Bank, Web based system, Donors, Administrators.

\section{INTRODUCTION}

In today's rapid developing scientific world technology has become a very important aspect of life. Today's generation is more depended on advanced technology than any other aspect. Today, most of the people use advance technologies in their daily life like Internet, Smartphone. So, the idea mentioned in this paper will make the process of blood bank less time consuming. In these application there will modules for donor, doctor, and blood bank. Donor has to register himself to use this improved system. For doctor, no need to call in every blood bank to check the blood availability. In improved system only doctor can check. Blood bank will send notification to donor regarding Donation camps or Emergency donation.

The blood bank management information system offers functionalities to quick access to donor records collected from various parts of the country. It enables monitoring of the results and performance of the blood donation activity such that relevant and measurable objectives of the organization can be checked. They are providing the efficient search who needs the blood in their own city as fast as possible. Blood donation and transfusion is an un-ignorable part of today's modern medicine and health care. Blood management has been considered as a challenging task because of life threatening nature of blood products. A very punctual administration is must due to its rapid perishable nature and required timely processing of blood and its related components. E-blood bank is an integrated blood bank automation system. The main purpose of E-blood bank is to interconnect all the blood banks of the state into a single network, validation, storage and circulation of various live data and information by using computation technology. Such system is able to assemble all the data of each and every individual into legible reports to support decision making from effective donor screening to optimal blood dissemination in the field.

The data which is stored on the computing devices will help the public for easy access to the blood availability status in blood banks on finger tips so that he can place a request or notify particular blood group in nearby blood bank(Especially rare groups) save a valuable life.

The blood bank system consists of various connected blood centers which store, distribute and collect human blood and hospital blood banks charging of transfusion related services. Given the life threatening nature of blood and blood components, it maintains the entire record of monitoring, controlling and the complete documentation of the whole procedure from blood collection to the blood storage and issuing the blood to the individuals in need. It is also responsible for the process of extracting the various categories like WBC, platelets. However during this process of updating and maintaining the data and information on the daily intervals, various errors which are impossible to avoid lead to significant risks in the mentioned procedure of blood donation and transfusion service. Errors at the time of administration of blood or blood components and sometimes the wrong entry of blood groups are the most frequent documented site of error this leads to storage or accumulation of wrong data in the records. Blood is considered as a very important aspect that sustains life. Blood is very precious and it saves many lives around the world in variety of circumstances. A blood bank is basically a place where the collected blood and the blood products are stored. The term blood bank refers to a place where the stored blood is properly tested to reduce the risk of transfusion events. Large refrigerated containers hold these blood products at a constant temperature and are dispersed wherever required. There are various blood banks available which carry out the similar process.

\section{RELATED WORK}

The blood bank system is mentioned above works on manual system. The blood bank provides unique ID to donors to keep track of records of donor as well as to retrieve the donor's information if required in future. If a person is in need of emergency blood first the doctor contacts the blood bank to make sure the required category of blood stock is available and informs the person to visit the blood bank to get the required category of blood. This process is very time 
consuming as time is very important factor due to the rapid perishable nature of blood and certain cases require blood within less time during such critical situations. Moreover the records are manually maintained in large catalogue due to this if a certain Person visits the blood bank again it is very time consuming to search his id in large catalogue.

The existing application does not provide facility of GPS, so in case if the user is new to a particular city it causes a problem to search the nearby blood banks. The main advantage of improved system is that it provides global positioning system which helps to identify the nearby blood banks in case of emergency.

The new application will be faster than the existing one. There will be some new or advance features in application. At basic level, the developed system is applicable for single blood bank but it can be extended to state or country. As most of the people use Android Smartphone so it will easily available to the people and also easy to use. The website for the application will be beneficial as those who don't have Smartphone they can use website. Doctor will never call in any blood bank as he can see available storage from different blood banks on his mobile or computer itself. The use of GPS will advance and fasten the searching technique. The contact between donor, doctor and blood bank will be fast.

\section{BLOOD DONORSHIP}

Potential donors are evaluated for anything that might make their blood unsafe to use.[3] The screening includes testing for diseases that can be transmitted by a blood transfusion, including HIV and viral hepatitis.[3] The donor must also answer questions about medical history and take a short physical examination to make sure the donation is not hazardous to his or her health. How often a donor can give varies from days to months based on what he or she donates and the laws of the country where the donation takes place.[3]

The collection of blood can be done manually or with automated equipment's that take only the various portions of blood that are required like platelets, WBC, RBC, plasma. This has led to an automatic need and interest in auto transfusion. Blood donations are divided into groups based on who will receive the collected blood. A 'directed' donation is when a person, often a family member, donates blood for transfusion to a specific individual. [3] Directed donations are relatively less when an established supply exists. In this case, family member of the recipient donates blood to replace the stored blood used in a transfusion, ensuring a consistent supply.[3] When a person had blood donation, blood that is used to make medications can be made from allergenic donations or from donations exclusively used for manufacturing.

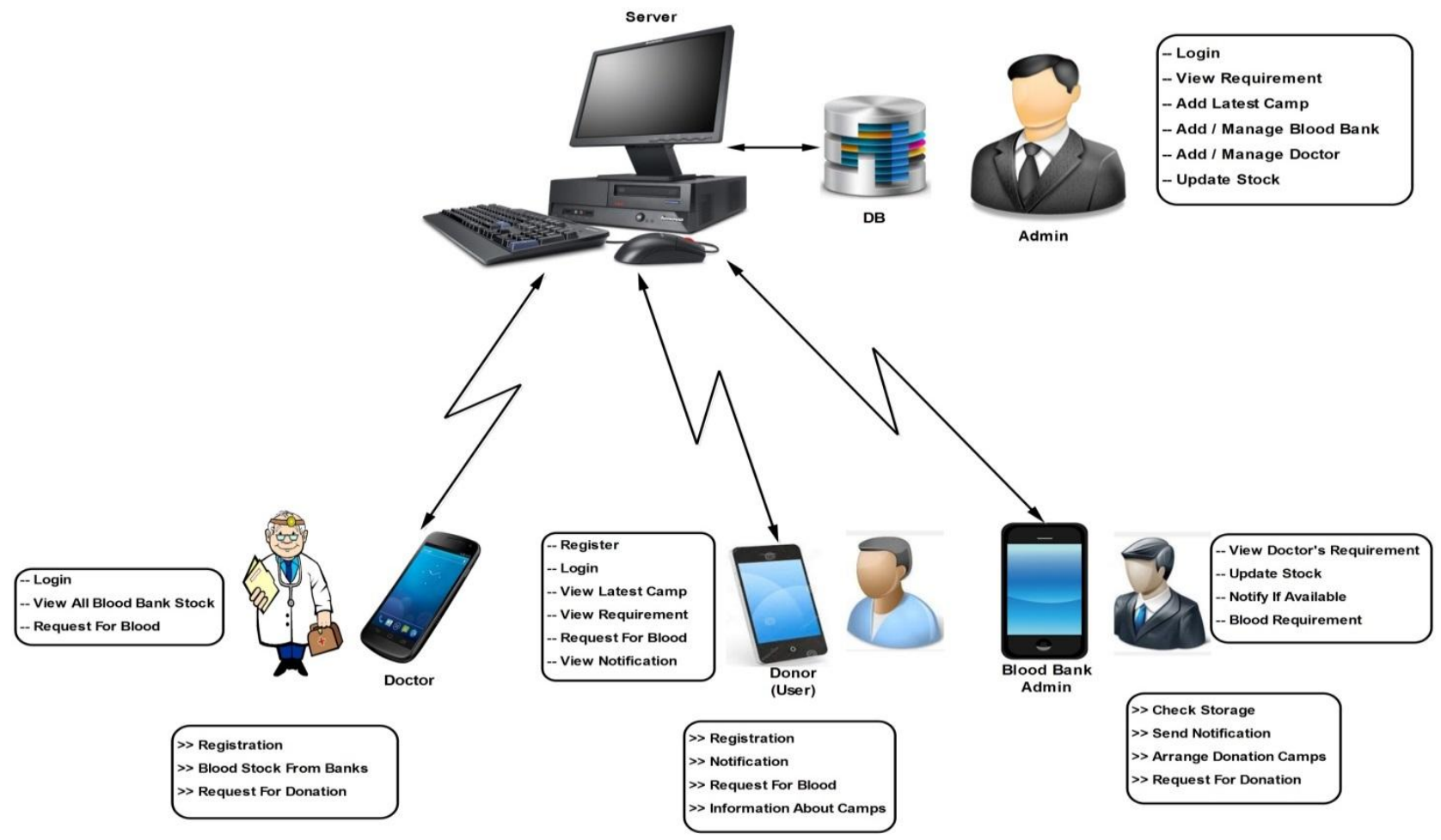

Fig1: Improved Blood Bank System.

\section{BLOOD TESTING}

1. The donor's blood type must be determined if the blood will be used for transfusions. The collecting agency usually identifies whether the blood is type A, $\mathrm{B}, \mathrm{AB}$, or $\mathrm{O}$ donor's $\mathrm{Rh}(\mathrm{D})$ type and will screen for antibodies to less common antigens.[3] More testing, including across match, is usually done before a transfusion. Group $\mathrm{O}$ is often cited as the "universal donor". but this only refers to cell transfusions. For plasma transfusions the system is reversed and $\mathrm{AB}$ is the universal donor type.[3]

2. False negatives are rare, but donors are discouraged from using blood donation for the purpose of anonymous STD screening because a false negative could mean a contaminated unit. The blood is usually discarded if these tests are positive, but there are some exceptions, such as antilogous donations. The donor is generally notified to the test result. 


\section{ACKNOWLEDGMENTS}

1. Our special thanks to Prof A.S.Shinde who contributed and gave a helping hand towards the development of Survey paper.

2. We would like to express our gratitude towards Prof. A.S.Shinde for their kind co-operation and encouragement.

\section{CONCLUSION}

In today's world of technology and communication where the world is heading towards the rapid growth and development. The increase in knowledge of mankind has certainly changed the gap of time interval as compared to ancient times which required plenty amount of time to carry out the same work. So this is our decent effort to reduce the gap between the need or requirement of blood and the immediate availability of blood in timely manner. This would turn out to be a life saviour application which would reach out to all the needy persons in time of emergency and would be a very successful application. Today the world is heading towards a Global Transformation where everything is online and today's generation has successfully adopted this culture so it won't be difficult to accept improved system and they would be comfortable using these improved system. It will prove as one more step to serve mankind. The improved system reduces the time required to collect and issue of blood process. Donor will get himself registered through these improved system. In case of emergency requirement the blood donor can place a request.

The blood bank can notify the donor in case of emergency. The blood bank collects the blood from different blood banks and he will inform if less. The application also provides the information about different event's so that the user will get information about the organized donation camps. The future scope of improved system is creating a huge network of blood banks spread over an entire country. The use of the Global Positioning System to identify the nearby blood banks. The system also inherits an algorithm that calculates how much blood stock will be required to fulfill the shortage of blood within the particular time span of a year.

\section{REFRENCES}

[1] Blood Bank Management Information System in India.Vikas Kulshreshtha Research Scholar ,Dr.Sharad Maheshwari Associate Professor Government Engineering College Jhalawar.Vikas Kulshreshtha,Dr.Sharad maheshwari, /International Journal of Engineering Research and Applications (IJERA) ISSN: 2248-9622.

[2] The Optimization of Blood Donor Information and Management System by Technopedia ,P. Priya1, V. Saranya2, S. Shabana3, Kavitha Subramani4 Department of Computer Science and Engineering, Panimalar Engineering College, Chennai, India International Journal of Innovative Research in Science, Engineering and Technology .Feburary 2014.

[3] Blood http://en.wikipedia.org/wiki/Blood_donation

[4] "Towards "digital blood-banking"", ITU-T Kaleidoscope: Innovations for Digital Inclusions Spyropoulos, B., Botsivaly, M., Tzavaras, , A., Spyropoulou, P., 2009. K-ID I2009. 